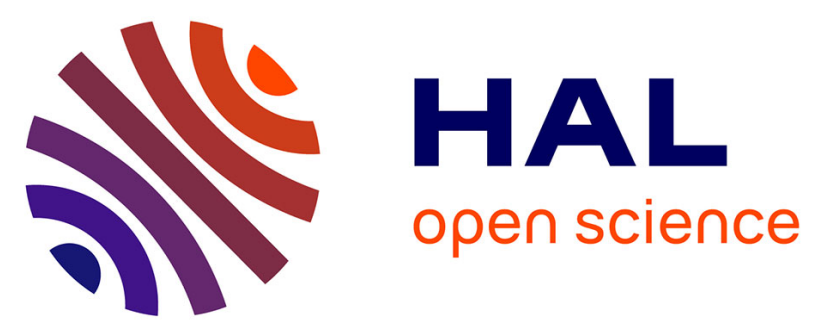

\title{
Comptes rendus des exposés présentés au 3e colloque d'Aussois sur les matériaux supraconducteurs et les applications de la supraconductivité. Aussois, 4-6 Avril 1973 patronné par la Société Française de Physique
}

\author{
G. Fournet
}

\section{To cite this version:}

G. Fournet. Comptes rendus des exposés présentés au 3e colloque d'Aussois sur les matériaux supraconducteurs et les applications de la supraconductivité. Aussois, 4-6 Avril 1973 patronné par la Société Française de Physique. Revue de Physique Appliquée, 1973, 8 (4), pp.393-396. 10.1051/rphysap:0197300804039300 . jpa-00243702

HAL Id: jpa-00243702

https://hal.science/jpa-00243702

Submitted on 1 Jan 1973

HAL is a multi-disciplinary open access archive for the deposit and dissemination of scientific research documents, whether they are published or not. The documents may come from teaching and research institutions in France or abroad, or from public or private research centers.
L'archive ouverte pluridisciplinaire HAL, est destinée au dépôt et à la diffusion de documents scientifiques de niveau recherche, publiés ou non, émanant des établissements d'enseignement et de recherche français ou étrangers, des laboratoires publics ou privés. 


\section{COMPTES RENDUS DES EXPOSÉS}

présentés au

\section{$3^{\text {e }}$ COLLOQUE D'AUSSOIS \\ SUR LES MATÉRIAUX SUPRACONDUCTEURS ET LES APPLICATIONS \\ DE LA SUPRACONDUCTIVITÉ}

AUSSOIS, 4-6 Avril 1973

patronné par

LA SOCIÉté FRANÇAISE DE PHYSIQUE

AVANT-PROPOS

Comme les précédents, le $3^{\mathrm{e}}$ Colloque d'Aussois a permis de réunir les chercheurs et les industriels intéressés par les supraconducteurs et leurs applications. Les principaux enseignements que l'on peut tirer de ce colloque sont les suivants :

1) activité toujours présente de la recherche fondamentale (matériaux hétérogènes, électrodynamique des vortex, nouveaux mécanismes conduisant à la supraconductivité...),

2) progrès sur l'élaboration des matériaux supraconducteurs,

3) progrès sur les « composites » qui les utilisent (un millier de brins supraconducteurs dans un fil de $0,5 \mathrm{~mm}$ de diamètre),

4) affirmation de l'avenir industriel des supraconducteurs : principalement les alternateurs de très grande puissance,

5) élargissement du champ d'utilisation de l'effet Josephson.

Je tiens à signaler que ce colloque n'aurait pu avoir lieu sans l'aide de la DGRST que je remercie au nom de tous les participants.

G. FOURNET. 



\section{LISTE DES PARTICIPANTS}

MM. Adde, Institut d'Electronique Fondamentale, Bât. 220, Université Paris-XI, 91-Orsay.

Amiet, DRME, 26, bd Victor, 75996 Paris Armée.

Assayrenc, CRTBT-CNRS, av. des Martyrs, 38-Grenoble.

Baixeras, Laboratoire de Génie Electrique, LCIE, 33, av. du Général-Leclerc, 92260 Fontenay-aux-Roses.

Berthet, CGE, Laboratoires de Marcoussis, route de Nozay, 91-Marcoussis.

Bethoux, CRTBT, av. des Martyrs, 38-Grenoble.

Bojoly, CRTBT, av. des Martyrs, 38-Grenoble.

Boyer, Laboratoire de Génie Electrique, LCIE, 33, av. du GénéralLeclerc, 92260 Fontenay-aux-Roses.

Burger, Laboratoire de Physique des Solides, Bât. 510, Université de Paris XI, 91-Orsay.

Bronca, CEN, Saclay, Département Saturne, BP 2, 91190 Gifsur-Yvette.

Carbonell, L'Air Liquide, Centre d'Etudes Cryogéniques, Les Engenières, BP 15, 38-Sassenage.

Cardinne, L'Air Liquides, Centre d'Etudes Cryogéniques, Le Engenières, BP 15, 38-Sassenage.

Cheeke, CRTBT-CNRS, av. des Martyrs, 38-Grenoble.

Chevallier, Centre d'Etudes Nucléaires de Grenoble (DMGLEMM), av. des Martyrs, 38-Grenoble.

Creppy, Laboratoire d'Electricité, Faculté des Sciences, BP 347, 51062 Reims.

Croitoru, Electricité de France, Service ERMEL, 17, av. du Général-de-Gaulle, 92140 Clamart.

Crozat, Institut d'Electronique Fondamentale, Bât. 220, Faculté des Sciences, 91400 Orsay.

Cyrot, CNRS, Laboratoire de Magnétisme, av. des Martyrs, 38-Grenoble.

Delahaye, LCIE, 33, av. du Général-Leclerc, 92260 Fontenayaux-Roses.

Delaval, DRME, 26, bd Victor, 75996 Paris Armée, Division Physique des Solides.

Delon, CNRS, Service Aéronomie, BP 3, 91310 Verrières-leBuisson.

Delrieu, CEA, Service de Physique des Solides et Résonance magnétique, BP 2, 91190 Gif-sur-Yvette.

Desportes, CEN SACLAY, STIPE B 123, BP 2, 91190 Gif-surYvette.

Desserre, Centre d'Etudes Nucléaires, LEMM/DMG, Bât. C 1, av. des Martyrs, 38-Grenoble.

Doulat, Centre d'Etudes Nucléaires, av. des Martyrs, 38Grenoble.

$M^{\text {me }}$ Dobrosavljevic, Institut de Physique, Studentski trg 16/III, BP 57, Belgrade, Yougoslavie.

Dubois, Laboratoires de Marcoussis, route de Nozay, 91460 Marcoussis.

Dupart, LGEP-LCIE, 33, av. du Général-Leclerc, 92260 Fontenay-aux-Roses.

Duret, Institut d'Electronique Fondamentale, Université de Paris XI, 91405 Orsay.

Fortini, Université de Caen, Laboratoire de Physique du Solide, 14-Caen.

Février, Centre d'Etudes Nucléaires Saclay, BP 2, 91190 Gif-surYvette.

Fourastier, DGRST, 35, rue Saint-Dominique, 75-Paris 7e.

Fourcade, MICHELIN, 63-Clermont-Ferrand.

Fournet, LGEP-LCIE, 33, av. du Général-Leclerc, 92260 Fontenay-aux-Roses.
Favier, Ecole des Mines, Centre des Matériaux, 91-CorbeilEssonne.

Gayte, CEM, 55, av. Jean-Jaurès, 93350 Le Bourget.

Genevey, CEA, SACLAY-DSS, BP 2, 91190 Gif-sur-Yvette.

Gilabert, Faculté des Sciences, parc Valrose, 06-Nice.

Gilchrist, CRTBT-CNRS, rue des Martyrs, 38-Grenoble.

Girard, CEM, Laboratoire de Cryogénie, 55, av. Jean-Jaurès, 93350 Le Bourget.

De Gennes, Collège de France, av. Marcellin Berthelot, 75Paris $5^{\mathrm{e}}$.

Gourrier, Institut d'Electronique Fondamentale, Université de Paris XI, 91-Orsay.

Granger, INSA, Département de Physique, 35031 Rennes Cedex.

Guyon, Université de Paris VI, Laboratoire de Physique des Solides, Bât. 510, 91-Orsay.

Hairie, Laboratoire de Physique du Solide, Université de Caen, 14-Caen.

Hellegouarch, CEM, 37, rue du Rocher, 75-Paris $8^{\mathrm{e}}$.

Klein Ch., Faculté des Sciences, place Eugène-Bataillon, 34Montpellier.

Klein J., Laboratoire de Physique des Solides de l'ENS, Université de Paris VI, 4, place Jussieu, Tour 23, 75230 Paris Cedex 05.

Kuhn, CRTBT-CNRS, av. des Martyrs, 38042 Grenoble.

Lasbley, INSA, Département de Physique, 35031 Rennes Cedex.

Laurenceau, ESPCI, 10, rue Vauquelin, 75-Paris $5^{\mathrm{e}}$.

Léger, Université de Paris VI, Groupe de Physique des Solides de l'ENS, Tour 23, 4, place Jussieu, 75230 Paris Cedex 05.

Letellier, CGE, Laboratoires de Marcoussis, route de Nozay, 91-Marcoussis.

Lottin, CEN SACLAY, BP 2, 91190 Gif-sur-Yvette.

Mailfert, LCIE-LGEP, 33, av. du Général-Leclerc, 92260 Fontenay-aux-Roses.

Maldy, CGE, Laboratoires de Marcoussis, route de Nozay, 91-Marcoussis.

Mallie, CRTBT-CNRS, av. des Martyrs, 38-Grenoble.

Marti, Institut Laue-Langevin, rue des Martyrs, 38-Grenoble.

Mazuer, CRTBT-CNRS, rue des Martyrs, 38-Grenoble.

Nedellec, Laboratoire de Physique des Solides, Université de Paris XI, Bât. 510, 91-Orsay.

$M^{\text {lle }}$ Morisseau, ESPCI, Laboratoire d'Electricité Générale, 10 , rue Vauquelin, 75 -Paris $5 \mathrm{e}$.

Nemoz, CRTBT, rue des Martyrs, 38-Grenoble.

Noer, Université de Paris Sud, Laboratoire de Physique des Solides, Bât. 510, 91405 Orsay.

Noiray, Laboratoire de Physique du Solide, Faculté des Sciences, parc Valrose, 06-Nice.

Nordman, Centre d'Etudes Cryogéniques de l'Air Liquide, BP 15, 38360 Sassenage.

Parouty, Thomson-CSF, av. Jean-Jaurès, 02301 Chauny.

Pascal, Université de Paris Sud, Institut d'Electronique Fondamentale, Bât. 220, 91405 Orsay.

Paumier, Laboratoire de Physique du Solide, Université de Caen, Esplanade de la Paix, 14000 Caen.

Pech, LCIE-LGEP 33, av. du Général-Leclerc, 92260 Fontenay-aux-Roses.

$\mathbf{M}^{\mathrm{me}}$ Pellan, INSA, 20, av. des Buttes de Coësmes, 35031 Rennes Cedex.

Perot, CEN SACLAY-DSS, BP 2, 91190 Gif-sur-Yvette.

$\mathbf{M}^{\mathrm{me}}$ Petipas, Laboratoire RX, Faculté des Sciences, 76-MontSaint-Aignan. 
Pouillange, CEN SACLAY, Service EDAP, BP 2, 91190 Gifsur-Yvette.

Porrot, Centre d'Etudes Nucléaires de Grenoble, BP 85, 38041 Grenoble Cedex.

Priel, BNM, 21, rue Casimir-Périer, 75-Paris $7^{\mathrm{e}}$.

Prost, CEM, 37, rue du Rocher, 75-Paris $8^{\mathrm{e}}$.

Quintanilha, Physics Dept. University of the Witwatersrand Johannesburg (South Africa).

Racek, Ecole des Mines, Centre des Matériaux, 91-CorbeilEssonne.

Renard Michel, CNRS, rue des Martyrs, 38-Grenoble.

Renard Marc, Laboratoire de l'Accélérateur Linéaire, Bât. 200, Université Paris Sud, 91405 Orsay.

Renard J. C., Laboratoire de Marcoussis, CRCGE, route de Nozay, 91-Marcoussis.

Mlle Raffy, Laboratoire de Marcoussis, route de Nozay, 91Marcoussis.

Revelli, Université de Paris Sud, Bât. 510, Laboratoire de Physique des Solides, 91405 Orsay.

Rey, Centre d'Etudes Nucléaires de Grenoble, rue de Martyrs, 38-Grenoble.
Rocher, Laboratoires de Marcoussis, CRCGE, route de Nozay, 91460 Marcoussis.

Rolland, INSA, Département de Physique, 35031 Rennes Cedex.

Rosenblatt, INSA, 20, av. des Buttes de Coësmes, 35031 Rennes Cedex.

Shimamoto, CEN SACLAY, BP 2, 91190 Gif-sur-Yvette.

Soulie, CEN, SACLAY, BP 2, 91190 Gif-sur-Yvette.

Talalaeff, CNET, Dpt. CPM/PMT, 22301 Lannion.

Thorel, Institut Laue-Langevin, rue des Martyrs, 38-Grenoble.

Torre, CNRS, Service d'Aéronomie, BP 3, 91370 Verrières-leBuisson.

Toulouse, INSA, 20, av. des Buttes de Coësmes, 35031 Rennes.

Vernet, Institut d'Electronique Fondamentale, Université de Paris Sud, Bât. 220, 91405 Orsay.

Waysand, Laboratoire de Physique des Solides, Université de Paris Sud, Bât. 510, 91405 Orsay.

Witgenstein, CERN, 1211 Genève.

Zenatti, CENG, LETI/DINR, BP 85, Centre de Tri, 38041 Grenoble Cedex. 